\title{
Structural basis of stereoselectivity in Candida rugosa lipase-catalyzed hydrolysis of secondary alcohols
}

\author{
Tanja Schulz, Rolf D. Schmid, Jürgen Pleiss ${ }^{1}$
}

Institute of Technical Biochemistry, University of Stuttgart, Allmandring 31, D-70569 Stuttgart, Germany

\footnotetext{
${ }^{1}$ Correspondence to: $\quad$ Dr. Jürgen Pleiss

Institute of Technical Biochemistry

University of Stuttgart

Allmandring 31

D-70569 Stuttgart, Germany

Phone: (++49)-711-685-3191

Fax: $\quad(++49)-711-685-3196$

Email: Juergen.Pleiss@po.uni-stuttgart.de
} 


\section{$\underline{\text { Abstract }}$}

Lipases are widely applied catalysts for highly enantioselective resolution of chiral secondary alcohols. While stereopreference is determined predominantly by the substrate structure, stereoselectivity (enantioselectivity and diastereoselectivity) depends on atomic details of interactions between substrate and lipase. Experimentally obtained stereoselectivity and activity in the hydrolysis of butanoic acid esters of two secondary alcohols with two neighbouring stereocenters by Candida rugosa lipase have been investigated by computer-aided molecular modeling of tetrahedral substrate intermediates in complex with the lipase. Breakdown of this intermediate is considered to be the rate-limiting step. Sterical interactions of stereo isomers with the side chain of catalytic histidine led to different orientations of the imidazole. The distance $d\left(\mathrm{H}_{\mathrm{N \varepsilon}}-\mathrm{O}_{\text {alc }}\right)$ between $\mathrm{H}_{\mathrm{N} \varepsilon}$ of the imidazole side chain of catalytic histidine and the alcohol oxygen of the substrate was identified to correlate with the experimentally determined reactivity order of the four stereo isomers. Modelled distances $\mathrm{d}\left(\mathrm{H}_{\mathrm{N} \varepsilon}-\mathrm{O}_{\text {alc }}\right)$ were short $(\leq 1.8 \AA)$ for $R R$ stereo isomers, which were also experimentally found to be hydrolyzed most rapidly; distances $\mathrm{d}\left(\mathrm{H}_{\mathrm{N \varepsilon}}-\mathrm{O}_{\text {alc }}\right)$ were about $2 \AA$ for $S S$ and $S R$ stereo isomers, which were converted at similar rates but at lower rate than $R R$ stereo isomers; finally, distances $\mathrm{d}\left(\mathrm{H}_{\mathrm{N} \varepsilon}-\mathrm{O}_{\text {alc }}\right)$ for $S R$ stereo isomers were greater than $4 \AA$, in accordance with very slow conversion of $S R$ stereo isomers.

\section{Keywords:}

Candida rugosa lipase; enantioselectivity; diastereoselectivity; molecular modeling; secondary alcohol. 


\section{$\underline{\text { Introduction }}$}

There has been growing interest among organic chemists to utilize biotransformations to prepare enantiomerically pure chemicals. A widely employed class of hydrolytic enzymes are lipases (EC 3.1.1.3) due to their high enantioselectivity, activity and stability, and their broad substrate specificity [1]. Chiral secondary alcohols are a well investigated class of substrates [2], which are converted in lipase catalyzed kinetic resolution through hydrolysis, esterification and transesterification. Stereopreference of most secondary alcohols can be predicted by a general rule [3], which have been validated experimentally. The rule (Fig. 1a) assumes that enantiomeric discrimination of lipases is predominantly based on the relative size of substituents at the stereo center ( $\alpha$-selectivity).

Recently, the influence of $\beta$-selectivity toward secondary alcohols with two neighbouring stereocenters, which contain an additional stereo center at the $\beta$ carbon (Fig. 1b), have been investigated experimentally [4]. Enantioand diastereoselectivities in C. rugosa lipase catalyzed hydrolysis of the four stereo isomers of acetic acid esters of $\mathbf{1}$ and $\mathbf{2}$ were measured [4], and the reactivity pattern of the four isomers was determined: the $R R$ isomer was hydrolyzed most rapidly, followed by $S S$ and $R S$, and finally by the $S R$ isomer. Both substrates have the same reactivity pattern and diastereoselectivity, while substrate 2 has decreased enantioselectivity.

The rule (Fig. 1a) to predict stereopreference of secondary alcohols assumes that fast conversion depends on the configuration at the $\alpha$ stereo center. Therefore, one would expect stereo isomers with preferred configuration at the $\alpha$-position - the $R R$ and the $R S$ stereo isomers for substrates $\mathbf{1}$ and $\mathbf{2}$ - to be hydrolyzed more rapidly than stereo isomers $S S$ and $S R$. However, it was experimentally shown, that RS hydrolyzes slightly slower than SS stereo isomers. Therefore the rule does not hold for stereo isomers of secondary alcohols with two neighbouring stereocenters. Since classification of substituents according to this rule is not appropriate for substrates $\mathbf{1}$ and $\mathbf{2}$, a different classification of substituents has to be considered (Fig. 1c). The rule holds, however, for each enantiomeric pair separately, and correctly predicts stereopreference: $R R$ is preferred to $S S, R S$ is preferred to $S R$. While stereopreference is successfully predicted by the rule, reaction rates of stereo isomers and the quantitative degree of stereoselectivity - enantio- and diastereoselectivity - could not be predicted.

We have reported [5] a simple model which allows prediction of enantioselectivity of Pseudomonas cepacia lipase catalyzed conversion of secondary alcohols. The model is based on a single geometrical parameter of tetrahedral substrate-lipase intermediates of slow-reacting enantiomers - the distance $d\left(\mathrm{H}_{\mathrm{N \varepsilon}}-\mathrm{O}_{\text {alc }}\right)$ between the hydrogen atom $\mathrm{H}_{\mathrm{N} \varepsilon}$ of catalytic histidine and the alcohol oxygen of the substrate. Quantum-chemical methods [6] have identified the transfer of the hydrogen atom $\mathrm{H}_{\mathrm{N} \varepsilon}$ from catalytic histidine to the alcohol oxygen of the 
substrate and the breakdown of the tetrahedral intermediate as rate-limiting step in lipase catalyzed hydrolysis [7,8]. As short distances $d\left(\mathrm{H}_{\mathrm{N \varepsilon}}-\mathrm{O}_{\text {alc }}\right)$ is indicative of fast conversion, short distances $\mathrm{d}\left(\mathrm{H}_{\mathrm{N \varepsilon}}-\mathrm{O}_{\text {alc }}\right) \leq 2.0 \AA$ in complexes of the slow-reacting enantiomer correlate with low enantioselectivity, while large distances $d\left(\mathrm{H}_{\mathrm{N} \varepsilon^{-}}\right.$ $\left.\mathrm{O}_{\text {alc }}\right) \geq 2.2 \AA$ are observed for substrates which are converted at high enantioselectivity.

In this study, we extended the model to predict $\beta$-selectivity in hydrolysis of butanoic acid esters of secondary alcohols with two neighbouring stereocenters and transferred the model to a different lipase, the lipase from $C$. rugosa. Based on experimentally determined data of C. rugosa lipase catalyzed hydrolysis of substrates $\mathbf{1}$ and $\mathbf{2}$, the relevance of $\mathrm{d}\left(\mathrm{H}_{\mathrm{N} \varepsilon}-\mathrm{O}_{\text {alc }}\right)$ for the prediction of stereoselectivity was verified. 


\section{Methods}

\section{$\underline{\text { Hard- and Software }}$}

Molecular modeling studies were carried out on Silicon Graphics workstations Indigo2/R10000. The software for energy minimisation and molecular dynamics simulations was Sybyl 6.3 and Sybyl 6.5 (Tripos, St. Louis, MO) using the Tripos force field [9].

\section{Molecular Modeling}

The X-ray structure of the open conformation of inhibited C. rugosa lipase [10] in complex with (1R)-menthylhexyl phosphonate [11] was taken from the PDB [12] (entry 1LPO). Solvent molecules and the inhibitor were removed and butanoic acid esters of the four stereo isomers of substrates $\mathbf{1}$ and $\mathbf{2}$ were covalently docked in their tetrahedral intermediate structure (Fig. 2). Docking was guided by the substrate analogous inhibitor (1R)menthyl-hexyl phosphonate [11]. The oxyanion was oriented toward the oxyanion hole, and the tetrahedral carbon of the substrate was covalently linked to the side chain oxygen $\mathrm{O} \gamma$ of catalytic serine; thus, the substratelipase complex mimics the first transition state in lipase catalyzed hydrolysis of esters of secondary alcohols. The protonation state at the catalytic histidine and the partial charges of the catalytic serine, histidine and substrate were modified [13] as calculated by the semiempirical method MNDO94/PM3 [14]. The structures of the substrate-lipase complexes were refined by energy minimization, and subsequent molecular dynamics simulations in vacuo with constrained protein backbone were performed [5]. The substrate-lipase complexes were equilibrated in three intervals of $1 \mathrm{ps}$ at $5 \mathrm{~K}, 30 \mathrm{~K}$ and $70 \mathrm{~K}$, and 4 ps at $100 \mathrm{~K}$, followed by a production phase of $1 \mathrm{ps}$ at $100 \mathrm{~K}$. The step size was $1 \mathrm{fs}$ up to $30 \mathrm{~K}$ and $0.5 \mathrm{fs}$ for 70 and $100 \mathrm{~K}$. The non-bonded interaction cut-off was set to $8 \AA$, the coupling constant to $10 \mathrm{fs}$, and the dielectric constant to 1.0. Conformers were saved every $40 \mathrm{fs}$. An average structure was created by superimposing and averaging twenty-five substratelipase complex structures of the production phase. The results of averaged structures from simulations at $100 \mathrm{~K}$ were analyzed by measuring the catalytically important distance $\mathrm{d}\left(\mathrm{H}_{\mathrm{N} \varepsilon}-\mathrm{O}_{\text {alc }}\right)$. 


\section{$\underline{\text { Results }}$}

\section{Binding of substrates}

Secondary alcohols $\mathbf{1}$ and $\mathbf{2}$ have two substituents of different size at the $\alpha$ stereo center. For both substrates, the medium-sized substituent at the $\alpha$ stereo center is methyl $(\mathrm{M} \alpha)$. The large substituent at the $\alpha$ stereo center includes the $\beta$ stereo center; substituents at the $\beta$ stereo center are classified as medium-sized (M $\beta$ ) and large substituent (L). M $\beta$ is methyl for $\mathbf{1}$ and 2, L is phenyl or $m$-tolyl for $\mathbf{1}$ or $\mathbf{2}$, respectively (Fig. 1c).

Two spherical binding pockets $-\mathrm{BP} \alpha$ and BP $\beta$ - were identified (Fig. 3), which have an appropriate geometry to bind medium-sized substituents $M \alpha$ and $M \beta$ of $\mathbf{1}$ and $\mathbf{2}$. The binding site of $C$. rugosa lipase is further limited by two rigid stops composed of backbone atoms, which are situated above and below the two stereo centers.

For enantiomers $R R$ and $S S$ of both substrates, $\mathrm{M} \alpha$ and $\mathrm{M} \beta$ bind to $\mathrm{BP} \alpha$ and $\mathrm{BP} \beta$, respectively, and the hydrogen atoms at the stereo centers direct toward the rigid stops (Fig. 4a). Thus, both enantiomers have minimal repulsive interactions and are well stabilized. During molecular dynamics simulations, no major conformational changes occurred. For both substrates, complexation of most rapidly converted stereo isomer $R R$ results in a proper orientation of side chain imidazole of catalytic histidine, which is prerequisite for efficient catalysis. Slower conversion of SS isomer is due to a slight change in the orientation of the side chain imidazole of the catalytic histidine. This difference in side chain orientation results from different sterical interactions of active histidine with substituents at the $\alpha$ stereo center of the $R R$ and $S S$ enantiomers, which is caused by the inversion at the two stereo centers.

For both enantiomers of the enantiomeric pair $R S$ and $S R, \mathrm{M} \alpha$ binds to $\mathrm{BP} \alpha$, and the hydrogen atom at the $\beta$ stereo center directs toward BP $\beta$. Repulsive interactions of $\mathrm{M} \beta$ with either of two rigid stops occur. During molecular dynamics simulations, both stereo isomers show geometric fluctuations. $\mathrm{M} \beta$ of the $R S$ isomer interacts with a stop composed of backbone atoms including the catalytic histidine, and pushes the side chain imidazole away from its proper orientation. Strong sterical repulsion with backbone atoms of the residues forming BP $\beta$ occurs for the $S R$ isomers, and results in a complete reorientation of the substrates during the simulations. In the reoriented conformation (Fig. 4b), the hydrogen atom at the $\alpha$ stereo center directs toward $\mathrm{BP} \alpha$, and $\mathrm{M} \alpha$ directs toward BP $\beta$ without binding to it. Repulsive sterical interactions of the substituents at the $\beta$ stereo center occur. In addition, in this conformation the substrate showed large motions during the simulations, and pushed away side chain imidazole of catalytic histidine from its productive orientation.

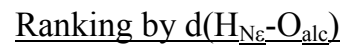


To quantify these visual observations, the catalytically important distance $d\left(\mathrm{H}_{\mathrm{N} \varepsilon}-\mathrm{O}_{\mathrm{alc}}\right)$ of the four stereo isomers was measured (Table 1) and compared with experimentally determined data. The $R R$ isomers had the shortest distance $(1.7 \AA$ and $1.8 \AA$ for $\mathbf{1}$ and $\mathbf{2}$, respectively), which correlates with experimentally observed reaction rates. The distances for $S S$ and $R S$ stereo isomers were similar $(2.0 \AA$ for $S S-\mathbf{1} / \mathbf{2}$ and $R S-\mathbf{2}, 2.1 \AA$ for $R S-\mathbf{1})$, which correlates to similar rates of conversion. Distances for $S R$ stereo isomers of 1 and 2 were d $\left(\mathrm{H}_{N \varepsilon}-\mathrm{O}_{\text {alc }}\right)>4$ $\AA$, thus preventing the formation of a hydrogen bond between $\mathrm{N} \varepsilon$ and $\mathrm{O}_{\text {alc }}$. This correlates to very slow conversion of $S R$ stereo isomers. Thus, ranking of the four stereo isomers by the modelled distance $d\left(\mathrm{H}_{\mathrm{N} \varepsilon}-\mathrm{O}_{\text {alc }}\right)$ correlates to experimentally determined reactivity orders of substrates $\mathbf{1}$ and $\mathbf{2}$.

\section{Activity of substrates 1 and 2}

Experimentally determined catalytic activity is in accordance with short distances $d\left(\mathrm{H}_{\mathrm{N} \varepsilon}-\mathrm{O}_{\text {alc }}\right)$ of experimentally most rapidly converted stereo isomers, which is isomer $R R$ for substrates $\mathbf{1}$ and 2 . Since the modelled distance $\mathrm{d}\left(\mathrm{H}_{\mathrm{N \varepsilon}}-\mathrm{O}_{\mathrm{alc}}\right)$ was slightly smaller for substrate $\mathbf{1}$ than for substrate $\mathbf{2}$, substrate $\mathbf{1}$ was predicted to be converted at higher rate. For a correct prediction of activity, all highly populated substrate conformations in the lipase binding site have to be considered. Two different orientations were observed for substrate 2: the $m$-methyl group at the tolyl substituent was situated at either of the two meta-positions. However, one of the two orientations of the $R R$ stereo isomer resulted in a non-productive hydrogen bond pattern (data not shown). Thus, both the shorter distance of $R R-\mathbf{1}$ and the population of two different conformations by $R R-\mathbf{2}$ - one with a active and one with a non-active hydrogen bond pattern - predict higher lipase activity toward substrate $\mathbf{1}$, which is in accordance with experiment. Different orientations of meta substituents in isomers $S S, R S$ and $S R$ of substrate 2 were also investigated, but did not influence the distances $\mathrm{d}\left(\mathrm{H}_{\mathrm{N} \varepsilon}-\mathrm{O}_{\mathrm{alc}}\right)$ for SS-2 and RS-2. For SR-2, only one orientation with a non-active hydrogen bond pattern was identified.

\section{Enantioselectivity}

Enantioselectivity is based on the ratio between lipase activity toward fast-reacting and slow-reacting enantiomers $\left(\mathrm{E}=\left(\mathrm{k}_{\mathrm{cat}} / \mathrm{K}_{\mathrm{m}}\right)_{\text {fast }} /\left(\mathrm{k}_{\mathrm{cat}} / \mathrm{K}_{\mathrm{m}}\right)_{\text {slow }}\right)$. Thus, high enantioselectivity results from large differences in activity, which should be reflected in large differences of $d\left(\mathrm{H}_{\mathrm{N \varepsilon}}-\mathrm{O}_{\mathrm{alc}}\right)$ between fast- and slow-reacting enantiomers. 
For the enantiomeric pair $R R$ and $S S$, the difference $\Delta \mathrm{d}\left(\mathrm{H}_{\mathrm{N} \varepsilon}-\mathrm{O}_{\mathrm{alc}}\right)=\mathrm{d}\left(\mathrm{H}_{\mathrm{N} \varepsilon}-\mathrm{O}_{\mathrm{alc}}\right)^{R R}-\mathrm{d}\left(\mathrm{H}_{\mathrm{N} \varepsilon}-\mathrm{O}_{\mathrm{alc}}\right)^{S S}$ are $0.3 \AA$ and 0.2 $\AA$ for substrate $\mathbf{1}$ and $\mathbf{2}$, respectively. The larger difference $\Delta \mathrm{d}\left(\mathrm{H}_{\mathrm{N \varepsilon}}-\mathrm{O}_{\mathrm{alc}}\right)$ for substrate $\mathbf{1}$ corresponds to a higher enantiomeric excess for $R R / S S-1$ (e.e. $62 \%$ ) than for $R R / S S-2$ (e.e. $35 \%$ ).

For the enantiomeric pair $R S$ and $S R$, the difference $\Delta \mathrm{d}\left(\mathrm{H}_{\mathrm{N \varepsilon}}-\mathrm{O}_{\mathrm{alc}}\right)$ is $2.2 \AA$ and $2.1 \AA$ for substrate $\mathbf{1}$ and $\mathbf{2}$, respectively. This agrees with experimentally determined slightly higher enantiomeric excess for $R S / S R-1$ (e.e. $59 \%$ ) than for $R S / S R-2$ (e.e. $43 \%$ ).

\section{$\underline{\text { Diastereoselectivity }}$}

Diastereoselectivity was estimated by the ratio of distances $d\left(\mathrm{H}_{\mathrm{N} \varepsilon}-\mathrm{O}_{\mathrm{alc}}\right)$ between enantiomeric pairs $\left(\left[\mathrm{d}\left(\mathrm{H}_{\mathrm{N} \varepsilon^{-}}\right.\right.\right.$ $\left.\left.\left.\mathrm{O}_{\mathrm{alc}}\right)_{R R}+\mathrm{d}\left(\mathrm{H}_{\mathrm{N \varepsilon}}-\mathrm{O}_{\mathrm{alc}}\right)_{S S}\right] /\left[\mathrm{d}\left(\mathrm{H}_{\mathrm{N \varepsilon}}-\mathrm{O}_{\mathrm{alc}}\right)_{R S}+\mathrm{d}\left(\mathrm{H}_{\mathrm{N} \varepsilon}-\mathrm{O}_{\mathrm{alc}}\right)_{S R}\right]\right)$. This ratio was determined as 0.6 for both substrates, thus indicating similar diastereoselectivities. The prediction of the model agreed with experimentally determined diastereoselectivities of $66 \%$ for 1 and $65 \%$ for 2 . 


\section{$\underline{\text { Discussion }}$}

The geometrical parameter $\mathrm{d}\left(\mathrm{H}_{\mathrm{N \varepsilon}}-\mathrm{O}_{\text {alc }}\right)$ allows substrate ranking and a qualitative interpretation of experimentally determined activities of C. rugosa lipase toward substrates $\mathbf{1}$ and 2, and modelling of relative enantio- and diastereoselectivities of the two substrates.

\section{Prediction of stereopreference}

Stereopreference toward chiral secondary alcohols has been reliably predicted by an empirical rule (Fig. 1a), which assumes that enantiomeric discrimination is predominantly based on the structure of the secondary alcohol moiety [3] ( $\alpha$-selectivity). The rule holds also for secondary alcohols with two neighbouring stereocenters $\mathbf{1}$ and 2, if stereopreference is predicted for each enantiomeric pair separately. However, the rule provides no information about stereopreference and reaction rates of the four stereo isomers (ranking), and enantio- and diastereoselectivity are not predicted. Thus, to gain insight in atomic details of interactions which mediate stereopreference, reaction rates, enantio- and diastereoselectivity of substrates $\mathbf{1}$ and $\mathbf{2}$ ( $\alpha$ - and $\beta$-selectivity), it is not sufficient to consider only structural properties of the substrate, but also interactions between the binding site of the biocatalyst and the substrates have to be considered within the model. Therefore, we investigated the first tetrahedral intermediates in the lipase-catalyzed hydrolysis of $\mathbf{1}$ and $\mathbf{2}$, since the breakdown of this intermediate is considered to be the rate-limiting step in lipase-catalyzed hydrolysis [6].

\section{$\underline{\text { Docking }}$}

Alcohol moieties include several rotatable bonds (degrees of freedom), and therefore the conformational space of the alcohol moiety is large. However, flexibility is restricted due to two well defined binding pockets of the substrate binding site: the hydrophobic tunnel, which accommodates the butanoic acid chain; M $\alpha$ and $\mathrm{M} \beta$, which bind the medium-sized substituents at the $\alpha$ and $\beta$ stereo center; two rigid stops, which consist of backbone atoms and limit the size of the binding site.

Comparison of X-ray structures (open conformations) of free [15] (PDB entry 1crl) and inhibited C. rugosa lipase [12] (PDB entry 1lpm) has shown that backbone atoms do hardly move upon substrate binding (Ca r.m.s. $0.2 \AA$ ), and thus follow the lock and key model [16], while side chains reorientate and show induced fit behaviour [17]. Accordingly, we performed molecular dynamics simulations by constraining the position of backbone atoms and allowing the side chains and the substrate to move. 
Tetrahedral intermediates of stereo isomers differed in the orientation of substituents in the binding site of $C$. rugosa lipase, resulting in different sterical and physico-chemical substrate-lipase interactions and therefore differences in the distance $\mathrm{d}\left(\mathrm{H}_{\mathrm{N \varepsilon}}-\mathrm{O}_{\text {alc }}\right)$. The high flexiblity of side chain imidazole of the catalytic histidine is in accordance with X-ray structures of inhibited C. rugosa lipase complexed with either enantiomer of menthyl hexylphosphonate [12], and with our model for P. cepacia lipase [5], where slow-reacting enantiomers in both cases distorted the orientation of the imidazole ring of catalytic histidine and broke the hydrogen bond between imidazole and the oxygen of the alcohol oxygen of the substrate, thus preventing efficient catalysis of slowreacting enantiomers and promoting high enantioselectivity.

\section{Prediction of activity and stereoselectivity (enantio- and diastereoselectivity)}

Quantum-chemical methods [18,19] have identified the first tetrahedral substrate-lipase complex and the distance $\mathrm{d}\left(\mathrm{H}_{\mathrm{N} \varepsilon}-\mathrm{O}_{\text {alc }}\right)$ as critical parameter of catalytic activity. Since differences of lipase activity [20,21] toward two enantiomers and diastereomers results in experimentally observable enantioselectivity $\left(\mathrm{E}=\left(\mathrm{k}_{\text {cat }} / \mathrm{K}_{\mathrm{m}}\right)_{\text {fast }} /\right.$ $\left.\left(\mathrm{k}_{\mathrm{cat}} / \mathrm{K}_{\mathrm{m}}\right)_{\mathrm{slow}}\right)$ and diastereoselectivity $\left(\left[\left(\mathrm{k}_{\mathrm{cat}} / \mathrm{K}_{\mathrm{m}}\right)_{R R}+\left(\mathrm{k}_{\mathrm{cat}} / \mathrm{K}_{\mathrm{m}}\right)_{S S}\right] /\left[\left(\mathrm{k}_{\mathrm{cat}} / \mathrm{K}_{\mathrm{m}}\right)_{R S}+\left(\mathrm{k}_{\mathrm{cat}} / \mathrm{K}_{\mathrm{m}}\right)_{S R}\right]\right)$, respectively, the hydrogen-bonding network [6], especially the distance $d\left(\mathrm{H}_{\mathrm{N \varepsilon}}-\mathrm{O}_{\mathrm{alc}}\right)$, is expected to be relevant to activity and stereoselectivity.

Distances $\mathrm{d}\left(\mathrm{H}_{\mathrm{N \varepsilon}}-\mathrm{O}_{\mathrm{alc}}\right)$ of stereo isomers of $\mathbf{1}$ and $\mathbf{2}$ showed a correct substrate ranking and therefore a correct prediction of activity of fast- and slow-reacting stereo isomers. However, comparison of different substrates through kinetic studies [6] have demonstrated that differences in the conversion of enantiomers do not result from enhanced reactivity of fast-reacting enantiomers but from reduced reactivity of slow-reacting enantiomers. Thus, the distance of fast-reacting stereo isomers (considering enantiomeric pairs) $R R$ and $R S$ should be of less importance with regard to stereoselectivity. This is true for the enantiomeric pair $R S-S R$, where the distance $\mathrm{d}\left(\mathrm{H}_{\mathrm{N} \varepsilon}-\mathrm{O}_{\text {alc }}\right)$ is larger for stereo isomer $S R \mathbf{- 1}$ than for $S R-\mathbf{2}$, resulting in higher enantioselectivity of substrate $\mathbf{1}$. For the enantiomeric pair $R R$ - SS of substrate 1 and 2, slow-reacting enantiomers (SS-1/2) show the same distance $\mathrm{d}\left(\mathrm{H}_{\mathrm{N} \varepsilon}-\mathrm{O}_{\text {alc }}\right)$, however, substrate 1 shows higher enantioselectivity. This is due to a second highly populated binding mode of $R R-2$, caused by the meta-methyl group of the large substituent, which shows a nonproductive hydrogen bond pattern and therefore reduced activity of $R R-2$. The distance $d\left(\mathrm{H}_{\mathrm{N} \varepsilon}-\mathrm{O}_{\mathrm{alc}}\right)$ is an appropriate geometrical parameter to reproduce experimentally determined activity and relative enantio- and diastereoselectivity in C. rugosa lipase catalyzed conversion of $\mathbf{1}$ and $\mathbf{2}$. 


\section{The model}

The model is based on structural properties of the first tetrahedral intermediate in lipase-catalyzed hydrolysis of butanoic acid ester of $\mathbf{1}$ and $\mathbf{2}$. As shown previously, docking methods are generally reliable and able to correctly predict stereopreference $[5,22,23]$. However, problems occur when energy-based scoring strategies are used to rank substrates [24]. To circumvent these limitations, our model uses common docking methods, while ranking is based on a new approach, a geometry-based scoring strategy.

Solvent can contribute significantly to activity and selectivity, but solvent engineering studies have shown that finding the best organic solvent for a lipase-catalyzed resolution is still a trial and error process, since there is no general rule to predict the best organic solvent. Our simulations of tetrahedral intermediates were performed in vacuo, and solvent effects were completely neglected. This model has previously been applied to P. cepacia lipase [5], where we observed a good correlation of the distance $d\left(\mathrm{H}_{\mathrm{N \varepsilon}}-\mathrm{O}_{\text {alc }}\right)$ to most solvent-optimized enantioselectivities. It seems that the maximum enantioselectivity which can be reached by solvent engineering is limited by the structure of biocatalyst and substrate. If this "structure-based enantioselectivity" is high and the optimal solvent is used, the two enantiomers are well separated by the lipase, while using sub-optimal solvent decreases enantioselectivity. However, if the "structure-based enantioselectivity" is low, solvent-engineering is not expected to be able to increase enantioselectivity.

This extension of our previous model, developed to investigate enantioselectivity of $P$. cepacia lipase toward chiral secondary alcohols [5], demonstrates that the model is transferable to different lipases as well as to different classes of substrates. The relevance of distance $d\left(\mathrm{H}_{\mathrm{N} \varepsilon}-\mathrm{O}_{\text {alc }}\right)$ to predict activity and stereoselectivity was verified. Further experimental data of lipase-catalyzed conversion of chiral secondary alcohols and other substrate classes will improve the model. Generalizing it to other lipases will help to understand common stereoselectivity-mediating factors and facilitate protein engineering studies. 


\section{$\underline{\text { References }}$}

[1] Schmid RD, Verger R (1998) Angew Chem Int Ed Engl 37: 1608-1633

[2] Kazlauskas RJ, Bornscheuer UT (1998) Biotransformations with Lipases. In: Rehm HJ, Reed G, Pühler A, Stadler PJW, Kelly DR (eds) Biotechnology, Vol.8a, Wiley-VCH, Weinheim, pp 37-191

[3] Kazlauskas RJ, Weissfloch ANE, Rappaport AT, Cuccia L (1991) J Org Chem 56: 2656-2665

[4] Angelis YS, Smonou I (1998) Tetrahedron Lett 39: 2823-2826

[5] Schulz T, Pleiss J, Schmid RD (2000) Protein Sci. 9: 1053-1062

[6] Ema T, Kobayashi J, Maeno S, Sakai T, Utaka M (1998) Bull Chem Soc Jpn 71: 443-453

[7] Chapus C, Sémériva M, Bovier-Lapierre C, Desnuelle P (1976) Biochemistry 15: 4980-4987

[8] Chapus C, Sémériva M (1976) Biochemistry 15: 4988-4991

[9] Clark M, Cramer RD III, van Opdenbosch N (1989) J Comp Chem 10: 982-1012

[10] Grochulski P, Young L, Schrag JD, Cygler M (1994) Protein Sci 3: 82-91

[11] Cygler M, Grochulski P, Kazlauskas RJ, Schrag JD, Bouthillier F, Rubin B, Serreqi AN, Gupta AK (1994) J Am Chem Soc 116: 3180-3186

[12] Bernstein FC, Koetzle TF, Williams GJB, Meyer EFJr, Brice MD, Rodgers JR, Kennard O, Shimanouchi T, Tasumi M (1977) J Mol Biol 112: 535-542

[13] Holzwarth HC, Pleiss J, Schmid RD (1997) J Mol Catal B: Enzymatic 3: 73-82

[14] Stewart JJP (1989) J Comp Chem 10: 209-220

[15] Grochulski P, Li Y, Schrag JD, Bouthillier F, Smith P, Harrison D, Rubin B, Cygler M (1993) J Biol Chem 268: 12843- 12847

[16] Fischer E (1894) Ber Dt Chem Ges 27: 2985-2993

[17] Koshland DE, Thoma JA (1960) J Am Chem Soc 82: 3329-3333

[18] Hu CH, Brinck T, Hult K (1998) Int J Quantum Chem 69: 89-103

[19] Monecke P, Friedemann R, Naumann S, Csuk R (1998) J Mol Model 4: 395-404

[20] Nakamura K, Kawasaki M, Ohno A (1996) Bull Chem Soc Jpn 69: 1079-1085

[21] Nishizawa K, Ohgami Y, Matsuo N, Kisida H, Hirohara H (1997) J Chem Soc, Perkin Trans 2: 1293-1298

[22] Scheib H, Pleiss J, Schmid RD (1998) Protein Eng 11: 675-682

[23] Scheib H, Pleiss J, Kovac A, Paltauf F, Schmid RD (1999) Protein Sci 8: 215-221

[24] Haeffner F, Norin T, Hult K (1998) Biophys J 74: 1251-1262 


\section{Figure captions}

Figure 1. (a) An empirical rule to predict the configuration of the fast-reacting enantiomer of chiral secondary alcohols in lipase-catalyzed conversions [3]; M, medium-sized substituent (e.g. methyl); L, large substituent (e.g. phenyl). (b) Classification of large and medium-sized substituents of secondary alcohols with two neighbouring stereocenters $\mathbf{1}$ and $\mathbf{2}$ in accordance to (a); stereo centers are labelled $\alpha$ and $\beta$. (c) Classification of medium-sized substituents $M \alpha$ and $M \beta$ at stereo centers $\alpha$ and $\beta$, respectively, and large substituent (e.g. Ar) at the $\beta$ stereo center.

Figure 2. Simplified binding site model of substrate-lipase complexes, including catalytically important residues and residues of the oxyanion-hole, and the first tetrahedral intermediate in lipase-catalyzed hydrolysis of secondary alcohols. The substrate is shown in bold; $\mathrm{R}$, fatty acid chain; $\mathrm{R}^{*}$ chiral alcohol moiety.

Figure 3. Three-dimensional binding site model of the lipase from C. rugosa in complex with the stereo isomer $R R-\mathbf{1}$. The two spherical binding pockets are labelled $\mathrm{BP} \alpha$ and $\mathrm{BP} \beta$; medium-sized substituents of $R R-\mathbf{1}$ are labelled $\mathrm{M} \alpha$ and $\mathrm{M} \beta$; the surface of side chain imidazole of catalytic histidine is colored in blue, and the position of $\mathrm{H}_{\mathrm{N} \varepsilon}$ and $\mathrm{O}_{\text {alc }}$ are indicated. Hydrogen atoms at $\mathrm{M} \alpha$ and $\mathrm{M} \beta$ and at the stereo centers $\alpha$ and $\beta$ are displayed and colored white; carbon atoms are colored grey, the alcohol oxygen $\mathrm{O}_{\text {alc }}$ is colored red; hydrogen atoms of the large substituent and the fatty acid chain are undisplayed.

Figure 4. Orientation of the four stereo isomers, $R R, S S, R S$ and $S R$, in the binding site of $C$. rugosa lipase: orientation of (a) the enantiomeric pair $R R-\mathbf{1} / \mathbf{2}$ and $S S-\mathbf{1} / \mathbf{2}$ and (b) of the second enantiomeric pair $R S-\mathbf{1} / \mathbf{2}$ and $S R-\mathbf{1} / \mathbf{2}$ as well as the reoriented conformation of $S R-\mathbf{1} / \mathbf{2}$ in the binding site of $C$. rugosa lipase. Arrows shaded in grey indicate repulsive sterical interactions; rigid stops ( $\equiv$ ) and binding pockets $\mathrm{BP} \alpha$ and $\mathrm{BP} \beta$ of the lipase are indicated; -Ar represents - phenyl or $m$-tolyl. 
Figure 1:

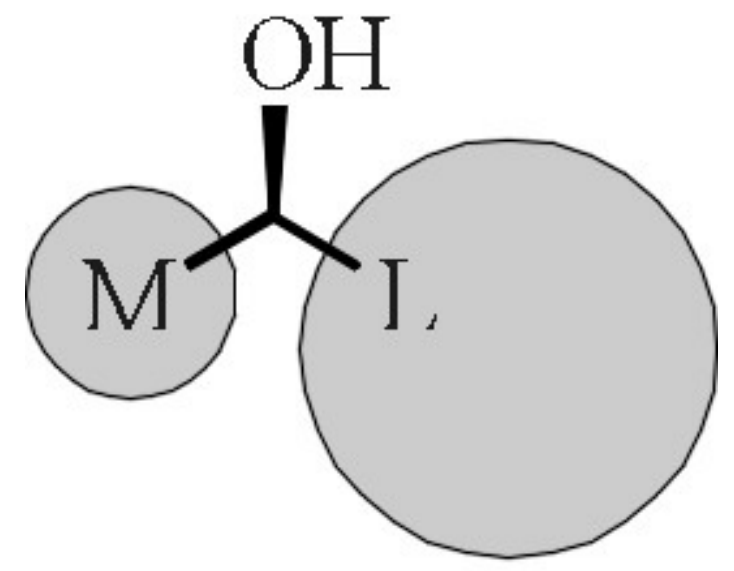

(a)

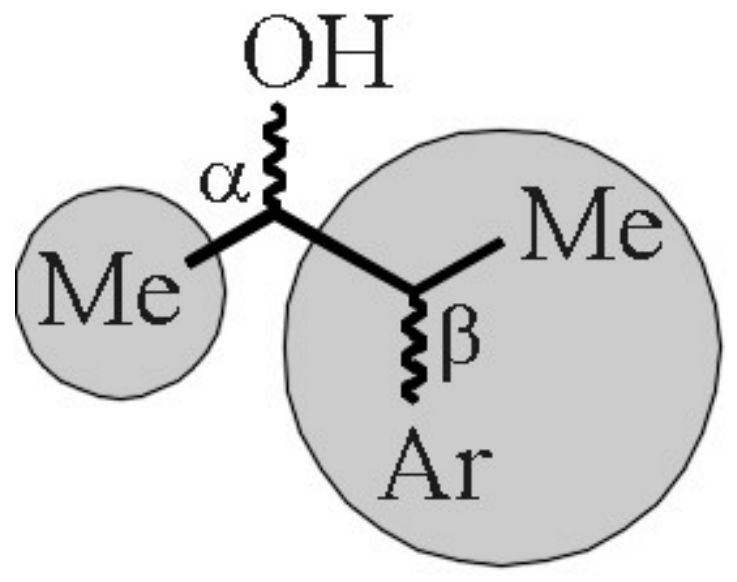

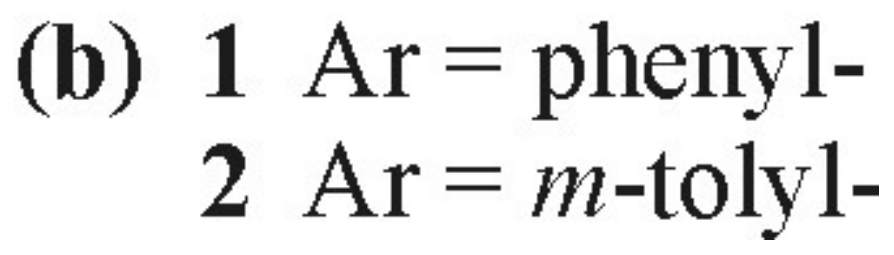




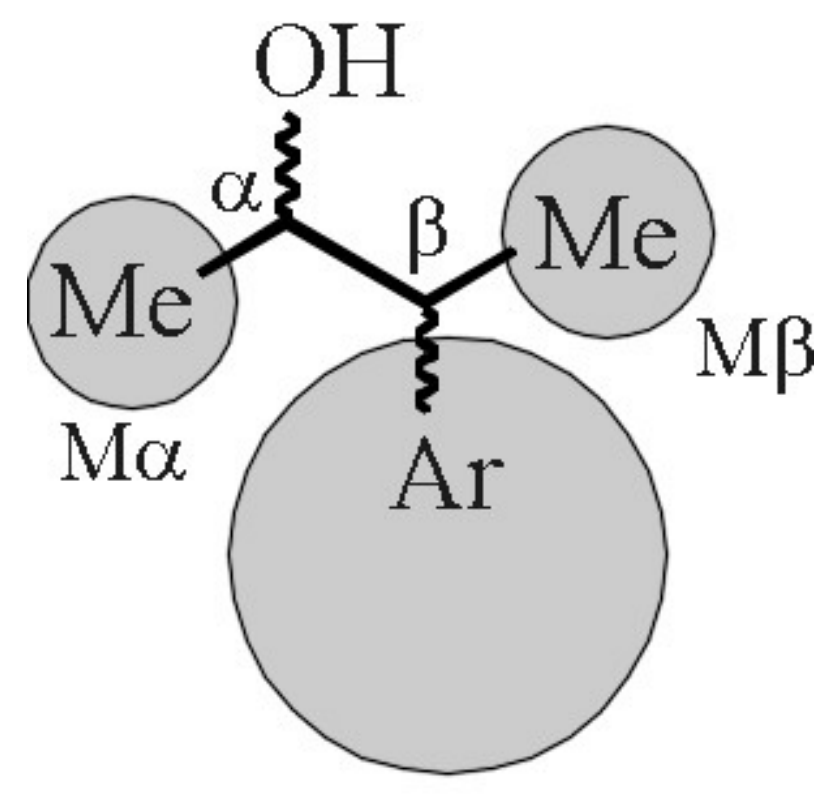

(c)

Figure 2:

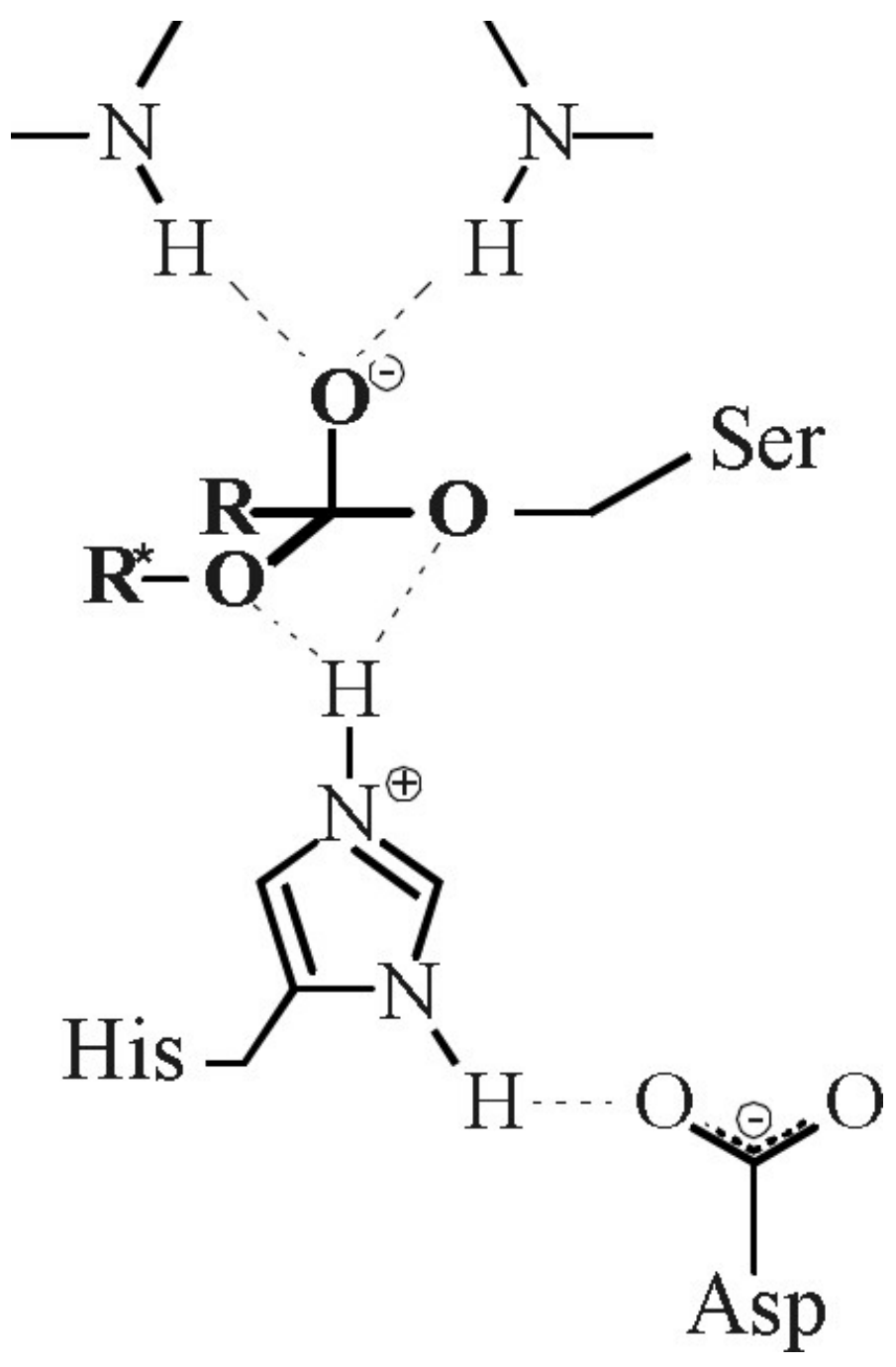


Figure 3:

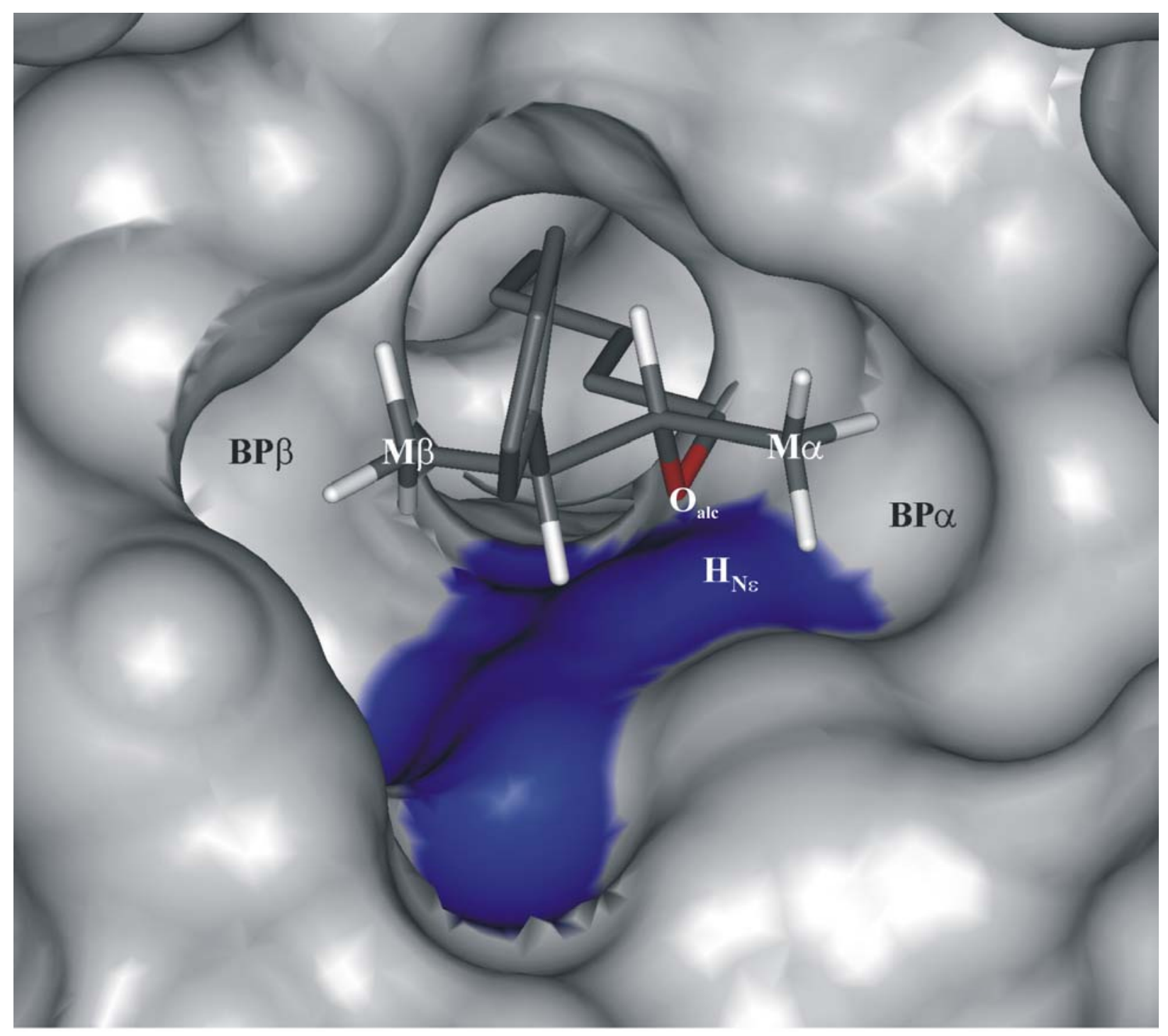

Figure 4:

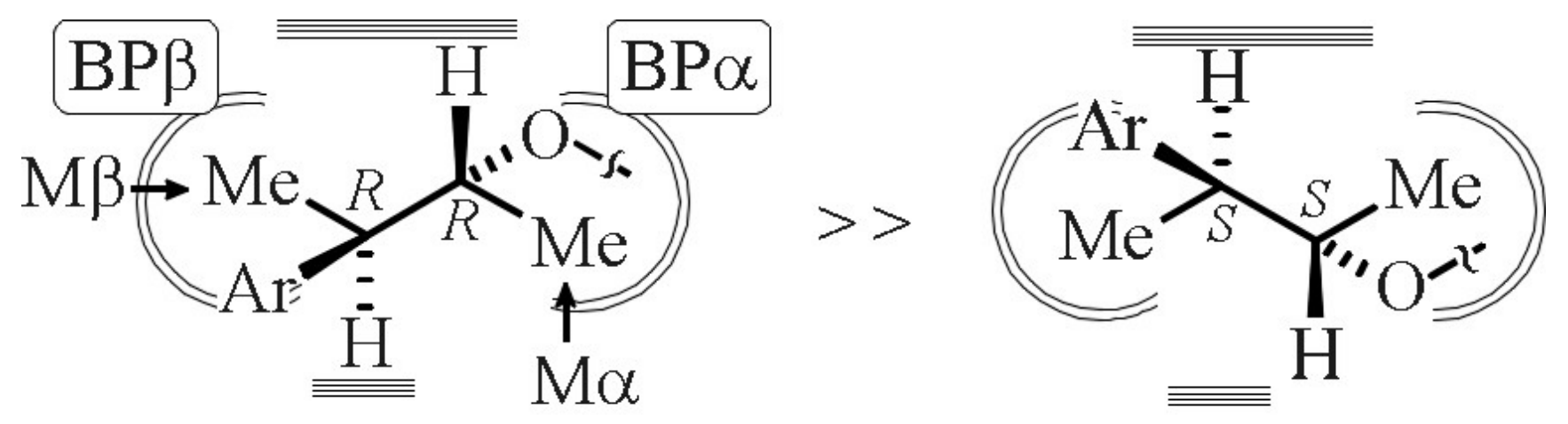

(a) 
(b)

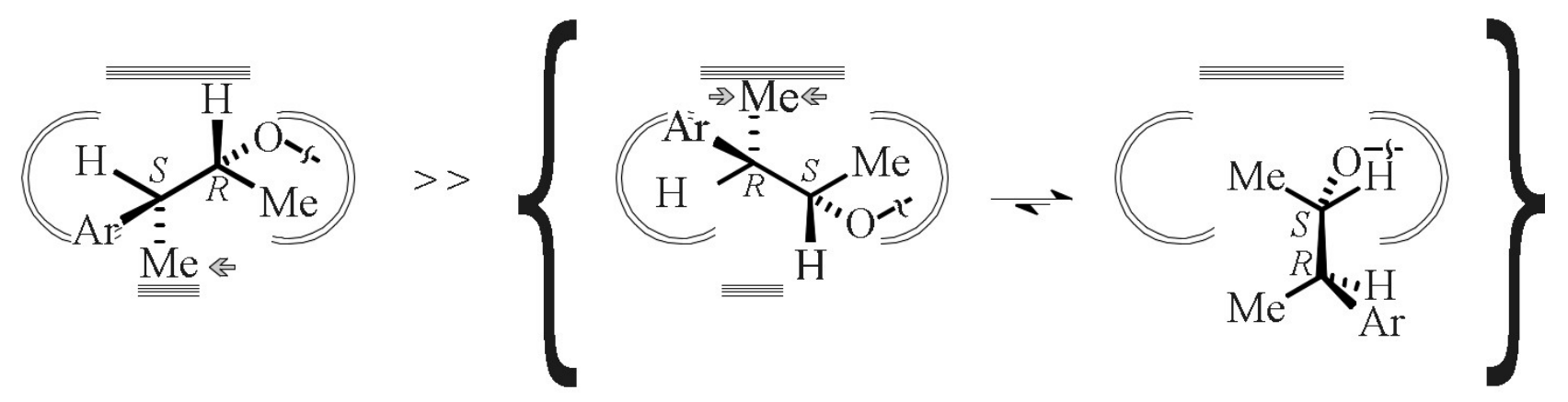

